\title{
Energy Budget of High-speed Plasma Flows in the Terrestrial Magnetotail
}

\author{
Yuduan Ma (马玉端 $)^{1,2}$, Jian Yang (杨建 $)^{1,2}$, M. W. Dunlop ${ }^{1,2,3}$, I. J. Rae ${ }^{4}$ (D), and Junying Yang (杨俊英 $)^{1,2}$ \\ ${ }^{1}$ School of Space and Environment, Beihang University, Beijing, 100039, People's Republic of China; ydma@buaa.edu.cn \\ ${ }^{2}$ Key Laboratory of Space Environment Monitoring and Information Processing, Ministry of Industry and Information Technology, Beijing, \\ People's Republic of China \\ ${ }^{3}$ Rutherford Appleton Laboratory, Oxfordshire, UK \\ ${ }^{4}$ Mullard Space Science Laboratory, University College London, Dorking, UK \\ Received 2019 April 1; revised 2020 March 17; accepted 2020 March 24; published 2020 April 30
}

\begin{abstract}
Significant advances have been presented for the theoretical model and quantitative investigation of the energy input from the solar wind and its subsequent release into the ring current, the Joule heating, and particle precipitation energy flux that are closely related to high-speed flows in the plasma sheet of the terrestrial magnetotail during both isolated and storm-time substorms. Here we further determine the plasma flow vorticity/ shear and heating observed in the terrestrial magnetotail by the Magnetospheric Multiscale (MMS) mission by deducing that other energy may be dissipated along with the above-mentioned three energy releases. The energy transported by the flow is also estimated here. Three-dimensional observations have shown new detailed information that can be compared with quasi-2D simulations. In this investigation, we calculate vorticity/shear directly from simultaneous observation of four MMS satellites. Our results generally indicate that plasma heating or temperature enhancements are related to both the flow vorticity/shear and current density, but more strongly with flow vorticity/shear. This research suggests that the ubiquitous energy input from the solar wind can be used to estimate plasma heating or temperature enhancements in the absence of any process or phenomenon relating to explosive energy release in planetary magnetospheres.
\end{abstract}

Unified Astronomy Thesaurus concepts: Plasma astrophysics (1261); Space plasmas (1544)

\section{Introduction}

The magnetosphere is a driven system powered by the energy early or directly input from the solar wind (e.g., Dungey 1961; Akasofu 1981; Du et al. 2011). Single and/or multisatellite observations in the magnetotail (e.g., Baumjohann et al. 1989; Angelopoulos et al. 1992, 1994; Cao et al. 2006, 2013) have demonstrated that the major transport of mass, energy, and magnetic flux in the plasma sheet occurs in a bursty fashion, associated with "bursty bulk flows" (BBFs; Angelopoulos et al. 1992). BBFs tend to occur more frequently during active times (e.g., Angelopoulos et al. 1994; Ma et al. 2009; Yu et al. 2017) but can also occur in the absence of substorms such as steady magnetospheric convection (SMC; e.g., Sergeev et al. 1996; Cao et al. 2008, 2010), convective bay (CB; e.g., Pytte et al. 1978), and even very quiet times (Beaujardière et al. 1994). BBFs often show typical characteristics of strong sunward and/or duskward flow bursts; some of them are accompanied by rapid increases of $B_{z}$ (dipolarization fronts) and strong duskward electric field (Schödel et al. 2001; Takada et al. 2006).

Plasma flow bursts observed at different locations of the plasma sheet and different magnetospheric activity levels (Baumjohann et al. 1989) can show quite remarkable localized intensity of the bulk speed (Kiehas et al. 2018) accompanied by flow shear (at least above $100 \mathrm{~km} \mathrm{~s}^{-1}$ ) relative to the slow and even stagnant ambient flow. Some of the bulk flows are characterized by a pronounced vortical or circulating motion (Keiling et al. 2009) in the magnetotail. Even a series of vortexlike plasma structures have been revealed in the dawnside plasma sheet using the Grad-Shafranov streamline reconstruction technique (Tian et al. 2010). Studies have suggested that the key difference between active- and quiet-time plasma sheets may be the integral amount of flux, plasma, and energy transported by these high-speed flows. The large-scale behavior of the magnetotail would then depend on the balance of the transport rates in the dayside and nightside magnetosphere (Sergeev et al. 1996).

A wide range of studies (e.g., Angelopoulos et al. 1992, 1994) have investigated the relationship between the energy transported by the Earthward BBFs and the energy increase of the ring current $\left(U_{\mathrm{R}}\right)$, the Joule heating $\left(U_{\mathrm{J}}\right)$, and particle precipitation energy flux $\left(U_{\mathrm{A}}\right)$ of both isolated and storm-time substorms based on parameterized empirical relations (e.g., Østgaard et al. 2002a, 2002b). Relatively little attention has been paid to the energy returned to the solar wind by tailward flows or plasmoid ejections from the tail reconnection (e.g., Baker et al. 1997; Fu et al. 2016). Even less attention has been paid to the energy dissipation like the plasma sheet heating associated with the fast flows observed by satellites (Baumjohann et al. 1989).

Braking of high-speed flow (e.g., Shiokawa et al. 1997; Fu et al. 2014; Liu et al. 2017) and associated dipolarizations for about 33\% of the BBF cases (Takada et al. 2006) indicated that flow energy may be transformed to electromagnetic energy (e.g., Keiling et al. 2009; De Spiegeleer et al. 2017; Liu et al. 2018) and/or may be converted to particle heating (e.g., Fu et al. 2013, 2019; Hamrin et al. 2014; Lu et al. 2017; Artemyev et al. 2018; Xu et al. 2018) as they propagate Earthward. Observations have shown that weakly collisional plasmas in planetary magnetospheres (e.g., Saur et al. 2002; Retinò et al. 2007) are typically more heated than what is expected from simplistic considerations (e.g., Sharma et al. 2012). In these plasmas, for the conversion of larger fluid scale energy into thermal degrees of freedom (e.g., Klimontovich 1997; Howes 2015), simulations have suggested that plasma flow vorticity (e.g., Huba 1996) and/ or shear (e.g., Markovskii et al. 2006; Yang et al. 2017a, 2017b; Del Sarto \& Pegoraro 2018) play a role in plasma heating. With 
hybrid and fully kinetic particle-in-cell simulations, Parashar \& Matthaeus (2016) demonstrated that protons are preferentially heated under one sign of vorticity and cooled in the opposite sense. However, there are a number of reports about bulk flows characterized by a pronounced vortical or circulating motion or vortex-like plasma structures in the magnetotail using the GradShafranov streamline reconstruction technique in the literature (e.g., Hones et al. 1978; Keiling et al. 2009; Tian et al. 2010). As shown in fully kinetic simulations, the pressure work could trigger individual energy conversion channels (for both ions and electrons) between fluid energy and random thermal energy (Yang et al. 2017a, 2017b). This idea has been tested in a few current layers by four satellites of the Magnetospheric Multiscale (MMS) mission (Pollock et al. 2016) of electron energy conversion channel (Chasapis et al. 2018) and local electron anisotropy. Direct calculation of the proton flow vorticity/shear using observations of flow fields from four satellites of MMS satellites (Pollock et al. 2016) in this paper.

The aim of this study is to investigate the energy budget for periods of the fast flows, including the energy input from the dayside, the energy increase of the ring current $\left(U_{\mathrm{R}}\right)$, the Joule heating $\left(U_{\mathrm{J}}\right)$, and particle precipitation energy flux $\left(U_{\mathrm{A}}\right)$, and energy dissipation like the plasma sheet heating related to flow vortices/shear and current enhancements (e.g., Huba 1996; Markovskii et al. 2006; Del Sarto et al. 2016; Parashar \& Matthaeus 2016). Plasma flow vortices/shear and current are directly calculated using the data from four satellites of MMS (Pollock et al. 2016). Examining the energy budget from the dayside to the magnetotail, ionosphere, ring current in large scale and ion heating in small scale, limited in hundreds of kilometers, will lead to better understanding on the balance of the transport rates in the dayside and nightside magnetosphere, and various evolution processes of fast flows for different magnetospheric activity levels (e.g., Sergeev et al. 1996; Parashar \& Matthaeus 2016).

\section{Instrumentation}

Data from the following instruments on board MMS were used in this study. The Fluxgate Magnetometer provides 3D magnetic fields with a cadence of 16 vectors per second in the fast survey mode (e.g., Russell et al. 2016). The Electric Double Probe provides 3D electric fields with a cadence of 32 vectors per second in the fast survey mode. The Fast Plasma Instrument provides 3D distributions for both ions and electrons with $4.5 \mathrm{~s}$ resolution in the fast survey mode. The energy ranges of the Fast Plasma Instrument are from $10 \mathrm{eV}$ to $30 \mathrm{keV}$ for both electrons and ions. Plasma moments (density, velocity, temperature, etc.) integrated by using the full distributions are also provided (Pollock et al. 2016).

In addition to the measurements of four MMS satellites in the near-Earth magnetotail, we use the AE and Dst index to investigate the energy budget by calculating the corresponding terms $U_{\mathrm{R}}, U_{\mathrm{J}}, U_{\mathrm{A}}$ with the geomagnetic data from http://wdc. kugi.kyoto-u.ac.jp/dstae/index.html and the higher-resolution solar wind data from https://omniweb.gsfc.nasa.gov/html/ ow_data.html. The Akasofu (1981) energy input parameter $\varepsilon$ is calculated using the OMNI data. It is well known that there is a time delay between energy input and the dissipation in the magnetosphere (Du et al. 2011; Xu et al. 2018). Time shifts of OMNI data to expected magnetosphere arrival times are done by the methods listed in https://omniweb.gsfc.nasa.gov/html/ ow_data.html\#time_shift.

\section{Overview of the Event}

Figure 1 shows the plasma and magnetic observations obtained from MMS3 during 02:16-03:36 UT on 2017 April 19. Ion energy spectra from the Fast Plasma Instruments (FPIs; Pollock et al. 2016) are shown in Figure 1(a). The ion energy spectra shown in Figure 1(a) are from FPIs for lower energies $(<30 \mathrm{keV})$. It is evident that the flux of low-energy ions below $1 \mathrm{keV}$ was less than that of the ions above $1 \mathrm{keV}$, so the ion density shown in Figure 1(b) is contributed mainly from ions up to $1 \mathrm{keV}$. The average ion density was about $0.75 \mathrm{~cm}^{-3}$ but varied from about 0.26 to $1.54 \mathrm{~cm}^{-3}$. Figure $1(\mathrm{c})$ shows the ion perpendicular (black) and parallel (red) temperatures. The average parallel temperature was about $4.35 \mathrm{keV}$, but the amplitude changed dramatically with a maximum of $11.44 \mathrm{keV}$ and a minimum of $0.75 \mathrm{keV}$. The situation of the perpendicular temperature was similar to that of the parallel one, except that the perpendicular temperature was smaller than the parallel one. Figures 1(d) and (e) depict the plasma flow moments and magnetic field components. The $(X, Y, Z)$ components of flows are shown, respectively, by blue, green, and red curves. Figure 1(d) shows that there were flow enhancements. The vertical dashed lines (red) show two time periods of the flow enhancements: from $t 1(02: 35: 34)$ to $t 2(02: 43: 27)$ and from $t 3$ (03:00:28) to t4 $(03: 26: 21)$ UT.

For the first flow enhancement, there were obvious increases in the three components of the bulk flow. In the $X$ component, the flow was weakly tailward with a peak at about $-146 \mathrm{~km} \mathrm{~s}^{-1}$ and relatively strongly Earthward with a peak at about $270 \mathrm{~km} \mathrm{~s}^{-1}$. In the $Y$ component, the flow was relatively strongly dawnward with a peak at about $-236 \mathrm{~km} \mathrm{~s}^{-1}$ and weakly duskward with a peak at about $130 \mathrm{~km} \mathrm{~s}^{-1}$. In the $Z$ component, the flow was comparably southward and northward with peaks at about -201 and $262 \mathrm{~km} \mathrm{~s}^{-1}$, respectively. For the second flow enhancement, there were clear increases in the three components of the bulk flow with a dominant $V_{x}$ component. In the $X$ component, the flow was weakly tailward with a peak at about $-265 \mathrm{~km} \mathrm{~s}^{-1}$ and strongly earthward with a peak at about $778 \mathrm{~km} \mathrm{~s}^{-1}$. In the $Y$ component, the flow was weakly dawnward with a peak at about $-197 \mathrm{~km} \mathrm{~s}^{-1}$ and strongly duskward with a peak at about $438 \mathrm{~km} \mathrm{~s}^{-1}$. In the $Z$ component, the flow was strongly southward with a peak at about $-345 \mathrm{~km} \mathrm{~s}^{-1}$ and weakly northward with a peak at about $142 \mathrm{~km} \mathrm{~s}^{-1}$. On the whole, the flow velocity oscillated in three components during the two periods of flow enhancements.

Three components of the magnetic field $\left(B_{x}\right.$ : blue; $B_{y}$ : green; $B_{z}:$ red) are shown in Figure 1(e). The relatively large negative value of $B_{x}$ and the small values of $B_{y}$ and $B_{z}$ indicated that MMS3 were in the outer parts of a fairly thick southern plasma sheet. During this time, MMS3 kept moving tailward from about $X \approx-9 R_{\mathrm{E}}$ to $-11 R_{\mathrm{E}}$ (with locations shown in the bottom of Figure 1), $B_{x}$ diminished gradually, $B_{y}$ varied slightly, and $B_{z}$ oscillated frequently. The plasma pressure shown in Figure 1(f) was mostly larger than $1 \mathrm{nPa}$ after $\mathrm{t} 1$ and increased with frequent oscillations. The magnetic pressure shown in Figure 1(g) decreased after $\mathrm{t} 1$ and decreased with frequent oscillations. The plasma beta was larger than unity with larger variations, even up to 4 after $\mathrm{t} 3$.

Given that there were large flow enhancements with a relatively large $V_{x}$ component of $\sim 800 \mathrm{~km} \mathrm{~s}^{-1}$ (Figure $1(\mathrm{~d})$ ), we suggest that the conditions in the terrestrial magnetotail were quite dynamic as a result of enhanced dayside solar wind driving. We then examine the solar wind and geomagnetic activities shown in Figure 2 during 0216-0336 UT. There was 


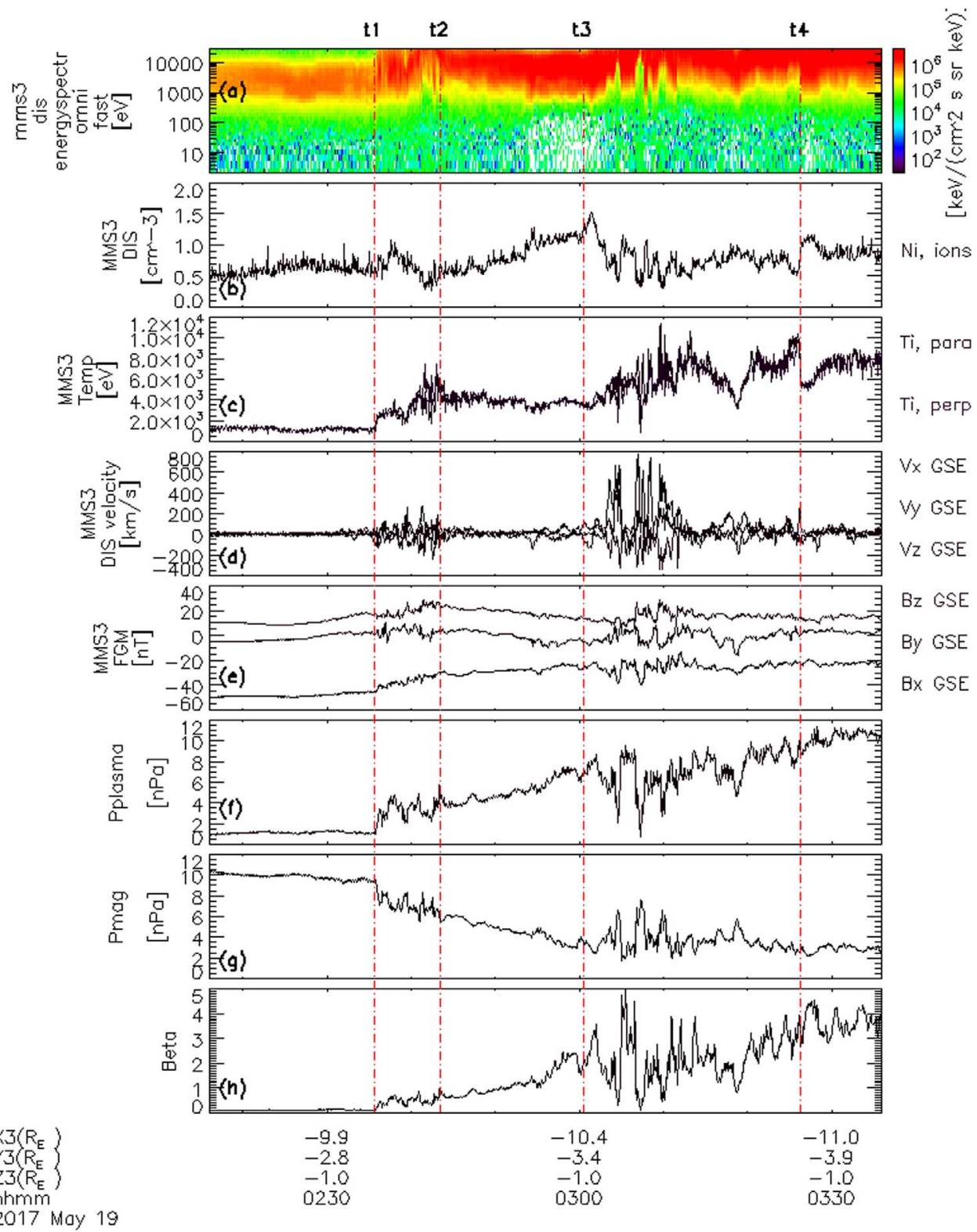

Figure 1. Magnetospheric observation from MMS3 spacecraft between 02:16 and 03:36 UT on 2017 April 19. Energy spectra from (a) ions obtained from FPIs. (b) Ion density. (c) Ion perpendicular (black) and parallel (red) temperatures. (d) $V_{X}$ (blue) $V_{y}$ (green) and $V_{z}$ (red) components of the ion flow. (e) $B_{x}$ (blue), $B_{y}$ (green), and $B_{z}$ (red) components of the magnetic fields. (f) Plasma pressure obtained by ion. (g) Magnetic pressure. (h) Plasma beta is calculated using plasma pressure obtained by ion and the magnetic pressure. The vertical dashed lines (red) show two time periods of the flow enhancements: from t1 (02:35:34) to t2 (02:43:27) and from t3 $(03: 00: 28)$ to $t 4(03: 26: 21)$ UT.

a gradual increase in the solar wind density from about 5 to $21 \mathrm{~cm}^{-3}$ shown in Figure 2(a); a similar trend also manifested in the dynamic pressure shown in Figure 2(e). The solar wind speed was above average at about $426 \mathrm{~km} \mathrm{~s}^{-1}$, and the interplanetary magnetic field (IMF) was mostly northward. The magnitudes of the $Y$ component and the $X$ component of the interplanetary magnetic field were comparable; their averaged absolute values were about 5 and $7 \mathrm{nT}$, respectively. The magnitude of the $Z$ component was small, with averaged absolute values less than $3 \mathrm{nT}$. The IMF kept northward for most of the time and turned southward only after 0334 UT. The $\mathrm{AL}$ index started to drop, resulting in an enhancement of the AE index after 0229 UT. That activity peaked at AE of about $263 \mathrm{nT}$, which is not considered as very strong substorm activity. The ASYM-H and ASYM-D index was larger than $5 \mathrm{nT}$ throughout the period, indicating that there was no geomagnetic storm activity at those times. This event occurred at Dst index rising from $7 \mathrm{nT}$ at $2 \mathrm{UT}$ to $19 \mathrm{nT}$ at $3 \mathrm{UT}$, with a minimum Dst index of $-24 \mathrm{nT}$ in the following $24 \mathrm{hr}$.

According to statistical results (e.g., Baumjohann et al. 1989; Angelopoulos et al. 1994; Ma et al. 2009), the high-speed flows are positively related to an increase of $\mathrm{AE}$ index. Combining the observations in Figures 1 and 2, we can find that the results obtained at these times from 02:16 and 03:36 on 2017 April 19 are different from the statistical trend. The observed enhancement of the flow in the near-Earth magnetotail was typical of the earthward high-speed flow braking region (Shiokawa et al. 1997), especially during the second flow enhancement period, which was accompanied by a peak at about $860 \mathrm{~km} \mathrm{~s}^{-1}$ of the flow velocity, with about $778 \mathrm{~km} \mathrm{~s}^{-1}$ in the earthward component. This resulted in a small AE index below $300 \mathrm{nT}$ and inspired us to investigate the possible energy 


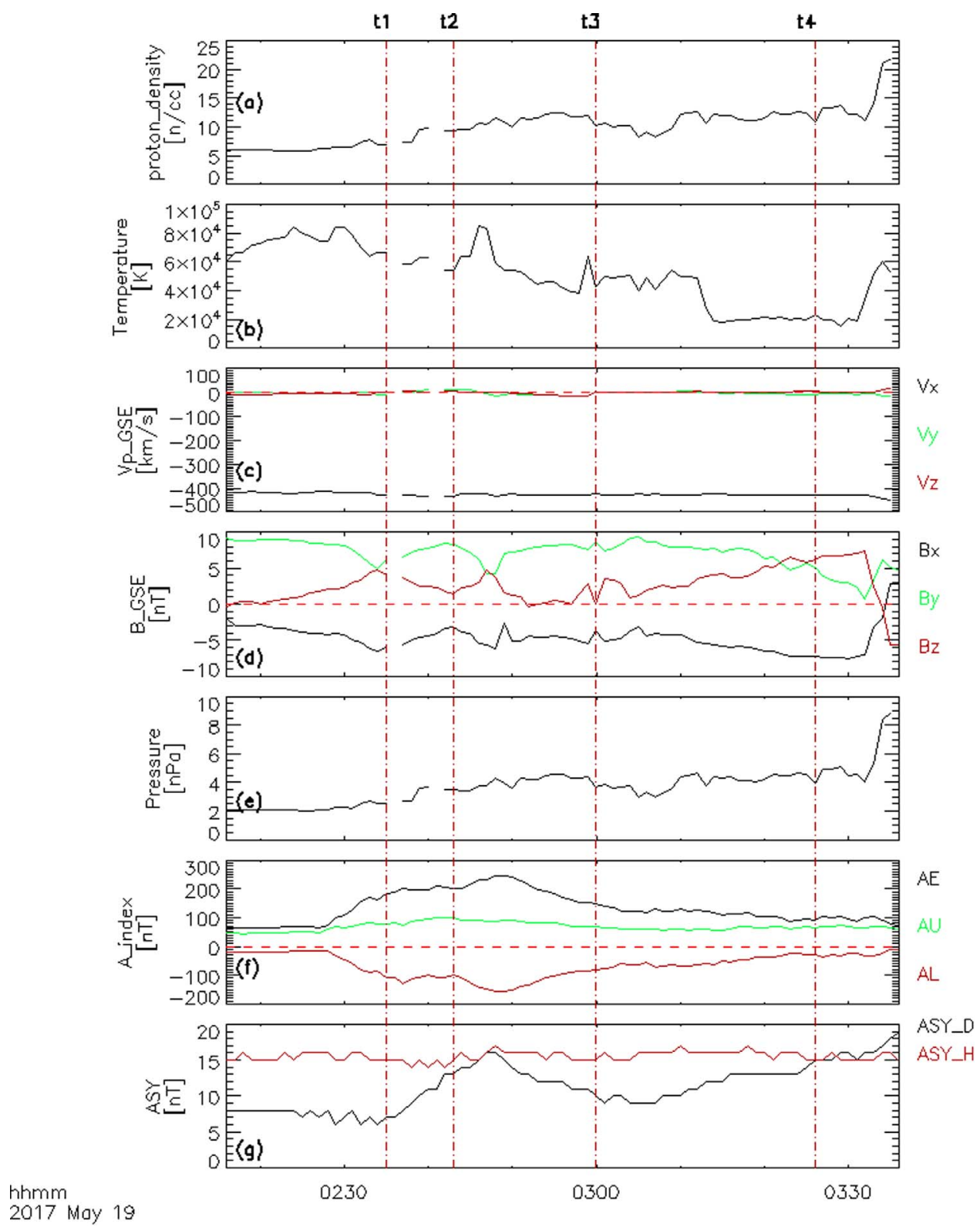

Figure 2. Solar wind and geomagnetic indices data from the OMNI data set between 02:16 and 03:36 on 2017 April 19. (a) Proton density. (b) Proton temperature. (c) Three components of the solar wind velocity ( $V_{X}$ : black; $V_{y}$ : green; $V_{z}:$ red). (d) $B_{x}$ (black), $B_{y}$ (green), and $B_{z}$ (red) components of the IMF magnetic field vector. (e) Dynamic pressure. (f) Auroral indices (AE: black; AU: green; AL: red). (g) ASYM-H (red) and ASYM-D (black) index.

transport and dissipation from the solar wind to the magnetosphere (Angelopoulos et al. 1994; Ma et al. 2009), ionosphere, and ring current based on parameterized empirical relations (Østgaard et al. 2002a, 2002b).

The energy flux density $Q$, done for simplicity in the framework of ideal MHD, can be expressed as follows (Parks 2004):

$$
\boldsymbol{Q}=\frac{\rho_{m} U^{2}}{2} \boldsymbol{U}+\frac{\gamma p}{\gamma-1} \boldsymbol{U}+\frac{(\boldsymbol{E} \times \boldsymbol{B})}{\mu_{0}}
$$

The terms on the right-hand side of Equation (1) represent the rate at which the kinetic energy of the fluid motion, the thermal energy, and the electromagnetic energy are being transported. Here $\rho_{m}$ is the mass density, $\boldsymbol{U}$ is the bulk velocity, $p$ is the thermal pressure, and $\gamma$ is the polytropic index. In the terrestrial plasma sheet, the polytropic index $\gamma$ is often assumed to be $5 / 3$ for simplicity, although the actual plasma sheet ions are not always adiabatic (Pang et al. 2015). Here $\boldsymbol{E}$ is the electric intensity, and $\boldsymbol{B}$ is the magnetic induction vector, $\boldsymbol{E}=-\boldsymbol{U} \times \boldsymbol{B}$. It is worth noting that the assumption $\boldsymbol{E}=-\boldsymbol{U} \times \boldsymbol{B}$ is valid only in the framework of ideal MHD and not rigorous. A more accurate extended MHD description would require Ohm's law. Since the average ion temperature versus the electron temperature $T_{i} / T_{e}$ was about 5.2 during the interval we studied, it was slightly below the narrow range of $5.5<T_{i} / T_{e}<11$ (Baumjohann et al. 1989). The electron pressure $P_{e}$ is also considered in this article.

Since Figure 1(c) indicates an important difference between temperatures parallel and perpendicular to the local magnetic field (sometimes by a factor $\sim 2$ ), we adopt a double polytropic closure such as $\gamma_{\perp}=2$ and $\gamma_{\|}=3$ (Hau \& Sonnerup 1993) to consider the contribution of the energy balance instead of assuming a standard polytropic closure of Equation (1) $\gamma=\frac{5}{3}$ 
(Angelopoulos et al. 1994). As presented by Hau et al. (1993) in their Equation (13), the double polytropic closure, $\gamma_{\perp}=2$ and $\gamma_{\|}=3$, implies no heat flux. We make this choice to correspond to a Chew-Goldberger-Low double-adiabatic closure. When we adopt $t \quad \gamma_{\perp}=2, \quad \gamma_{\|}=3$ (Hau \& Sonnerup 1993) to consider the contribution of the energy balance, energy flux density will be resolved into parallel and perpendicular to the magnetic field.

The terms on the right-hand side of Equation (1) representing various energies transported by the plasma flow in the parallel and perpendicular to the magnetic field can be resolved into Equations (2)-(4) shown as the following:

$$
\begin{aligned}
\boldsymbol{Q}_{\|} & =\frac{\rho_{m} U^{2}}{2} \boldsymbol{U}_{\|}+\frac{\gamma_{\|} p_{\|}}{\gamma_{\|}-1} \boldsymbol{U}_{\|}=\boldsymbol{Q}_{\boldsymbol{F}_{-} \text {parp }}+\boldsymbol{Q}_{\boldsymbol{P}_{-} \text {parp }} \\
& =\boldsymbol{Q}_{\boldsymbol{F} \boldsymbol{P} \_ \text {parp }}
\end{aligned}
$$

Here we denote the term $Q_{F-p a r p}$ as the component of the flow energy flux density parallel to the magnetic field, the term $Q_{P_{\text {_parp }}}$ as the component of the thermal energy flux density parallel to the magnetic field, and the term $Q_{F P}$ parp as the component of the sum of the flow dynamic and thermal energy parallel to the magnetic field.

$$
\begin{aligned}
\boldsymbol{Q}_{\perp 1} & =\frac{\rho_{m} U^{2}}{2} \boldsymbol{U}_{\perp}+\frac{\gamma_{\perp} p_{\perp}}{\gamma_{\perp}-1} \boldsymbol{U}_{\perp}=\boldsymbol{Q}_{\boldsymbol{F}-\text { perp }}+\boldsymbol{Q}_{\boldsymbol{P} \_ \text {perp }} \\
& =\boldsymbol{Q}_{\boldsymbol{F} \boldsymbol{P} \_ \text {perp }}
\end{aligned}
$$

Here we denote the term $Q_{F_{-} \text {perp }}$ as the component of the flow energy flux density perpendicular to the magnetic field, the term $Q_{P}$ _perp as the component of the thermal energy flux density perpendicular to the magnetic field, and the term $Q_{F P \text { _perp }}$ as the component of the sum of the flow dynamic and thermal energy perpendicular to the magnetic field, that is, perpendicular to $B$ aligned to $\boldsymbol{U} \times \boldsymbol{B}$.

$$
\boldsymbol{Q}_{\perp 2}=\frac{\boldsymbol{E} \times \boldsymbol{B}}{\mu_{0}}=\frac{(-\boldsymbol{U} \times \boldsymbol{B}) \times \boldsymbol{B}}{\mu_{0}}=\boldsymbol{Q}_{E B_{-} \text {perp }} .
$$

Here we denote the term $Q_{E B}$ _perp as the component of the Ponyting energy flux density that is perpendicular to both $B$ and $U$ aligned to $(-U \times B) \times B$.

If we assume that the flow areas parallel and perpendicular to the magnetic field are both $3 \times 3 R_{\mathrm{E}}^{2}$, absolute values of the terms in Equations (2)-(4) are shown in panels (a)-(c) of Figure 3.

Combining panels (a)-(c) of Figure 3 during the first period, we can see that the magnitude of flow energy transport density $Q_{F}$-parp was the smallest and could be as low as $1.2 \times 10^{12} \mathrm{erg} \mathrm{s}^{-1}$. For the component parallel to the magnetic field, $Q_{F-\text { parp }}$ is several times or even two orders of magnitude smaller than $Q_{P}$ parp. For the component parallel to the magnetic field, $Q_{F-\text { parp }}$ and $Q_{P_{-} \text {parp }}$ are several times or even two orders of magnitude smaller than those perpendicular to the magnetic field $Q_{F-\text { perp }}$ and $Q_{P \_ \text {perp. }}$. The Poynting flux density $Q_{E B \_ \text {perp }}$ was slightly larger than the thermal energy flux density $Q_{p \_ \text {perp }}$ for most of the time. For the component perpendicular to the magnetic field, the maximum energy transport density $Q_{F-\text { perp }}$ is about $7.2 \times 10^{16} \mathrm{erg} \mathrm{s}^{-1}$, which is below the expected BBF transport during "typical" substorm conditions in the ionosphere shown in Table 5 (Angelopoulos et al. 1994). The maximum energy transport density of $Q_{p \text { _perp }}$ is about $1.3 \times 10^{18} \mathrm{erg} \mathrm{s}^{-1}$, and the maximum energy transport density of $Q_{E B_{-} \text {perp }}$ is about $2.1 \times 10^{18} \mathrm{erg} \mathrm{s}^{-1}$, which are well above the expected BBF transport during "typical" substorm conditions of $4.0 \times 10^{17} \mathrm{erg} \mathrm{s}^{-1}$ including the ionosphere but slightly below that of $3.0 \times 10^{18} \mathrm{erg} \mathrm{s}^{-1}$ including the ring current shown in Table 5 (Angelopoulos et al. 1994).

Combining panels (a)-(c) of Figure 3 during the second period, we can see that the smallest magnitude of flow energy flux density $Q_{F}$ parp was about $5.8 \times 10^{11} \mathrm{erg} \mathrm{s}^{-1}$. However, the component parallel to the magnetic field, $Q_{F-\text { parp }}$, is larger than $Q_{F_{\text {_perp }}}$ for most of the time. The maximum of $Q_{F \_ \text {parp }}$ is about $7.7 \times 10^{17} \mathrm{erg} \mathrm{s}^{-1}$, which is almost twice the expected BBF transport during "typical" substorm conditions in the ionosphere shown in Table 5 (Angelopoulos et al. 1994). For the flow component parallel and perpendicular to the magnetic field, $Q_{F-\text { parp }}$ and $Q_{F-\text { perp }}$ are several times or even one order of thermal magnitude smaller than those of $Q_{P_{-} \text {parp }}$ and $Q_{P_{-} \text {perp. }}$. The Poynting flux density $Q_{E B}$ _perp was less than the thermal energy flux density $Q_{P_{-} \text {parp }}$ and $Q_{P_{-} \text {perp }}$ for most of the time. For the component perpendicular to the magnetic field, the maximum energy transport density $Q_{F-\text { perp }}$ could be as high as $6.0 \times 10^{17} \mathrm{erg} \mathrm{s}^{-1}$, which is also above the expected $\mathrm{BBF}$ transport of $4.0 \times 10^{17} \mathrm{erg} \mathrm{s}^{-1}$ during "typical" substorm conditions in the ionosphere shown in Table 5 (Angelopoulos et al. 1994). The maximum energy transport density of $Q_{E B}$ _perp could be as high as $2.3 \times 10^{18} \mathrm{erg} \mathrm{s}^{-1}$, which is slightly below the expected BBF transport of $3 \times 10^{18} \mathrm{erg} \mathrm{s}^{-1}$ during "typical" substorm conditions including the ring current (Angelopoulos et al. 1994). The maximum energy transport density of $Q_{p_{-} \text {parp }}$ could be as high as $4.6 \times 10^{18} \mathrm{erg} \mathrm{s}^{-1}$, which is even above the expected BBF transport of $3 \times 10^{18} \mathrm{erg} \mathrm{s}^{-1}$ during "typical" substorm conditions including the ring current (Angelopoulos et al. 1994).

By simultaneously taking into account the sign, the sums of the parallel and perpendicular components of the flow, thermal, and electromagnetic energy transport density are plotted in Figure 3(d), represented by $\left|Q_{F P_{-} \text {parp }}\right|$ (blue) parallel to the magnetic field, $\left|Q_{F P \_ \text {perp }}\right|$ (green) perpendicular to the magnetic field, and $\left|Q_{E B \_ \text {perp }}\right|$ perpendicular to both bulk flow vector and the magnetic field (red), respectively. The absolute peak energy flux density was $9.3 \times 10^{17} \mathrm{erg} \mathrm{s}^{-1}$ (of $\left|Q_{F P_{-} \text {parp }}\right|$ ), $3.4 \times 10^{18} \mathrm{erg} \mathrm{s}^{-1}$ (of $\left|Q_{F P \_ \text {perp }}\right|$ ), and $2.1 \times 10^{18} \mathrm{erg} \mathrm{s}^{-1}$ (of $\left|Q_{E B_{-} \text {perp }}\right|$ ) during the first period. That of the second period was $5.3 \times 10^{18} \mathrm{erg} \mathrm{s}^{-1}$ (of $\left|Q_{F P_{-} \text {parp }}\right|$ ), $6.3 \times 10^{18} \mathrm{erg} \mathrm{s}^{-1}$ (of $\left|Q_{F P \_ \text {perp }}\right|$ ), and $2.3 \times$ $10^{18} \mathrm{erg} \mathrm{s}^{-1}$ (of $\left.\mid Q_{E B \_ \text {perp }}\right)$ ), respectively. It can be seen that the energy transport peak was the largest it can be in the direction either parallel or perpendicular to the magnetic field; the energy transports in the direction perpendicular to both bulk flow vector and the magnetic field during the two periods were also quite large and cannot be ignored. Therefore, when we calculate the total energy transport density $U_{\mathrm{F}}$ of the plasma flow in Figure 3(e), the contributions of these three components are also taken into account, i.e., $\left|U_{\mathrm{F}}\right|=\left|Q_{F P \_ \text {parp }}+Q_{F P_{-} \text {perpr }}+Q_{E B_{-} \text {perp }}\right|$ as shown by the gray curve. Apart from the energy transported by the flows $U_{\mathrm{F}}$, the $\varepsilon$ (Equation (8) in Akasofu 1981) parameter from the solar wind (Akasofu 1979, 1981), the energy increase of the ring current $\left(U_{\mathrm{R}}\right.$, Equation (1) in Østgaard et al. 2002a), the Joule heating $\left(U_{\mathrm{J}}\right.$, Equation (10) in Østgaard et al. 2002a), and the particle precipitation energy flux $\left(U_{\mathrm{A}}\right.$, Equation (11) in Østgaard et al. $2002 \mathrm{~b}$ ) are estimated using the most appropriate parameterized methods in earlier studies and used to figure out the feature of the energy transfer, dissipation, and transport.

Taking into account that the data resolution of $U_{\mathrm{R}}, U_{\mathrm{J}}, U_{\mathrm{A}}$, and $\varepsilon\left(\mathrm{erg} \mathrm{s}^{-1}\right)$ is lower than that of $U_{\mathrm{F}}$, we use different symbols to represent the data points $U_{\mathrm{R}}$ (black star), $U_{\mathrm{J}}$ (blue plus sign), $U_{\mathrm{A}}$ (green triangle), and $\varepsilon$ (red diamond) in 


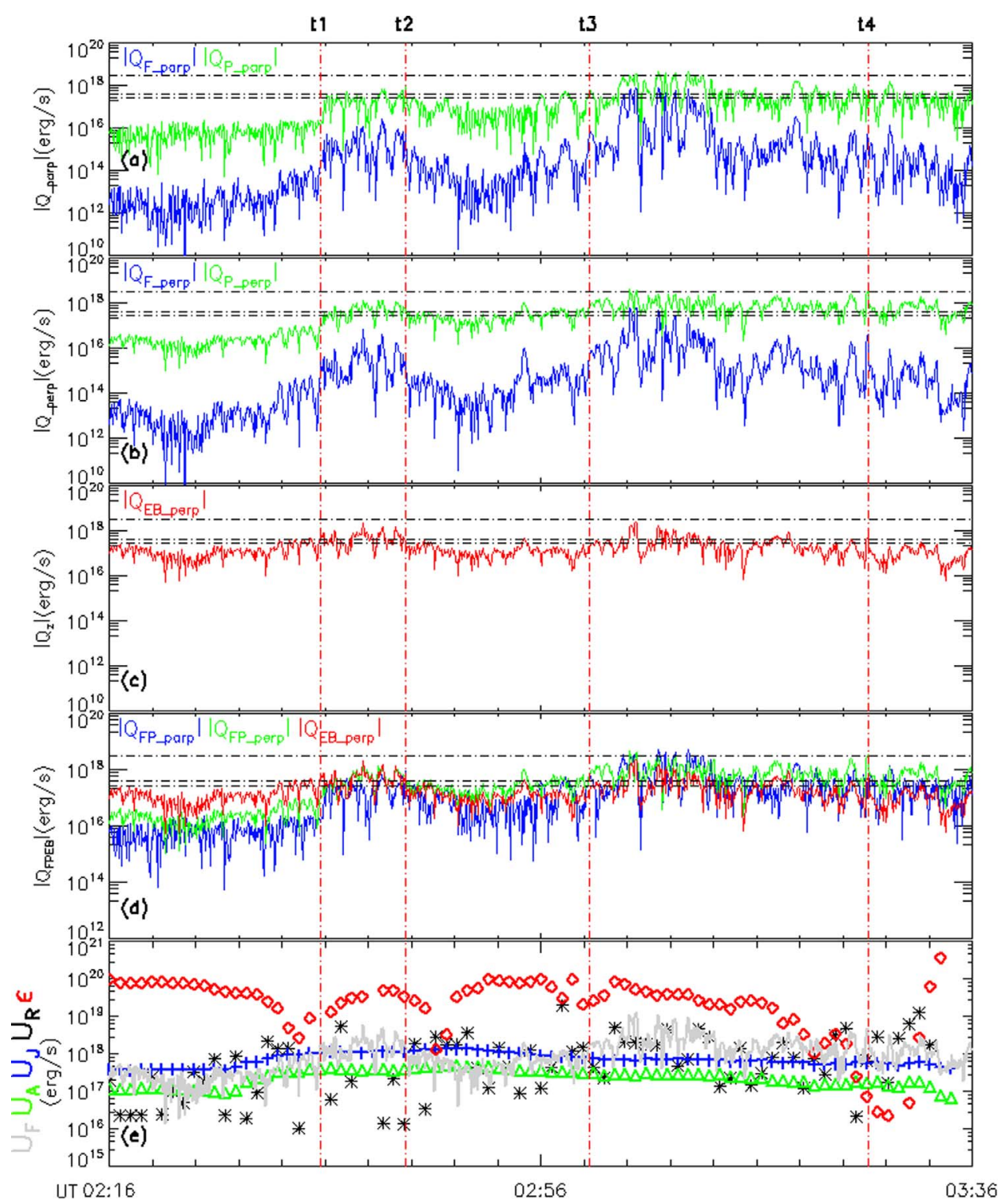

Figure 3. Absolute parallel to the magnetic field (a), perpendicular to the magnetic field (b), and perpendicular to both the magnetic field and bulk flow vector (c) component of various energy flux densities (erg s $\left.{ }^{-1}\right)\left|Q_{F-\text { parp }}\right|,\left|Q_{F \_ \text {perp }}\right|$ (blue), $\left|Q_{P_{-} \text {parp }}\right|,\left|Q_{P_{-} \text {perp }}\right|$ (green), $\left|Q_{E B_{-} \text {perp }}\right|$ (red), and the sum component of various energy flux densities (d) $\left|Q_{F P_{-} \text {parp }}\right|$ (blue), $\left|Q_{F P_{-} \text {perp }}\right|$ (green), $\left|Q_{E B_{-} \text {perp }}\right|$ (red) using FPI and FGM data from MMS3 between 02:16 and 03:36 UT on 2017 April 19. (e) Energy increase of the flow $U_{\mathrm{F}}$ (gray) including these three components, ring current $U_{\mathrm{R}}$ (black star), the Joule heating of the atmosphere $U_{\mathrm{J}}$ (blue plus sign), the precipitation for the total electron flux $(0.01-100 \mathrm{keV}) U_{\mathrm{A}}$ (green triangle), and the Akasofu energy input parameter $\varepsilon$ (red diamond) using solar wind and geomagnetic indices data from the OMNI data. The three horizontal dashed-dotted lines represent the values of $3.0 \times 10^{18} \mathrm{erg} \mathrm{s}^{-1}, 4.0 \times 10^{17} \mathrm{erg} \mathrm{s}^{-1}$, and $2.7 \times 10^{17} \mathrm{erg} \mathrm{s}^{-1}$, respectively, in Figure 3(a) from top to bottom. It is the same for Figures 3(b)-(d).

Figure 3(e). Both $U_{\mathrm{J}}$ and $U_{\mathrm{A}}$ were fairly constant, with the former larger than the latter. There are many variations of the terms $\varepsilon, U_{\mathrm{F}}$, and $U_{\mathrm{R}}$. It is evident that the peaks among all the terms appear in the term $\varepsilon$ for most of the time, while the troughs of the term $\varepsilon$ can be smaller than $U_{\mathrm{F}}, U_{\mathrm{J}}, U_{\mathrm{A}}$, and $U_{\mathrm{R}}$. Dramatic changes in the amplitude occurred in the term $U_{\mathrm{R}}$ with troughs and peaks among all the terms. The second most dramatic changes in amplitude occurred in the term $U_{\mathrm{F}}$, and there were some large variations in the term $U_{\mathrm{R}}$. The amplitude of $U_{\mathrm{F}}$ was mostly larger than that of $U_{\mathrm{A}}$. During the periods before $\mathrm{t} 1$, from $\mathrm{t} 2$ to $\mathrm{t} 3$, and after $\mathrm{t} 4$, the amplitude of $U_{\mathrm{F}}$ was mostly less than $U_{\mathrm{R}}$. Although the relative magnitude of $U_{\mathrm{F}}$ and $U_{\mathrm{R}}$ changed frequently, the amplitude of $U_{\mathrm{F}}$ was mostly larger than $U_{\mathrm{R}}$ in the periods from t1 to $\mathrm{t} 2$ and from $\mathrm{t} 3$ to $\mathrm{t} 4$. Except that $U_{\mathrm{J}}$ was always greater than $U_{\mathrm{A}}$, the relative amplitude of various energy flux density changed frequently from 0216 to
0336 UT. It is difficult to exactly conclude the features of the energy transport and dissipation as a whole. If we take a normalized value of 100 for $\varepsilon$, then the averaged absolute values of $U_{\mathrm{F}}, U_{\mathrm{R}}, U_{\mathrm{J}}$, and $U_{\mathrm{A}}$ in the periods from t1 to t2 were about 5.8, 3.6, 2.4, and 1.3, respectively.

The corresponding averaged absolute values in the periods from $\mathrm{t} 3$ to $\mathrm{t} 4$ were about $7.9,5.5,2.2$, and 0.8 , respectively, so the amplitude of these energy flux densities can be roughly ranked as $\varepsilon, U_{\mathrm{F}}, U_{\mathrm{R}} U_{\mathrm{J}}$, and $U_{\mathrm{A}}$ in the periods from $\mathrm{t} 1$ to $\mathrm{t} 2$ and from $\mathrm{t} 3$ to $\mathrm{t} 4$. This order of magnitude indicates that the energy density of high-speed flow transported may not be only dissipated in the energy increase of the ring current, the Joule heating, and particle precipitation energy flux. We suggest that there should be other energy dissipations during these periods. The obvious increase of the temperature as indicated by Figure 1(c) implies that part of the energy may be dissipated to 

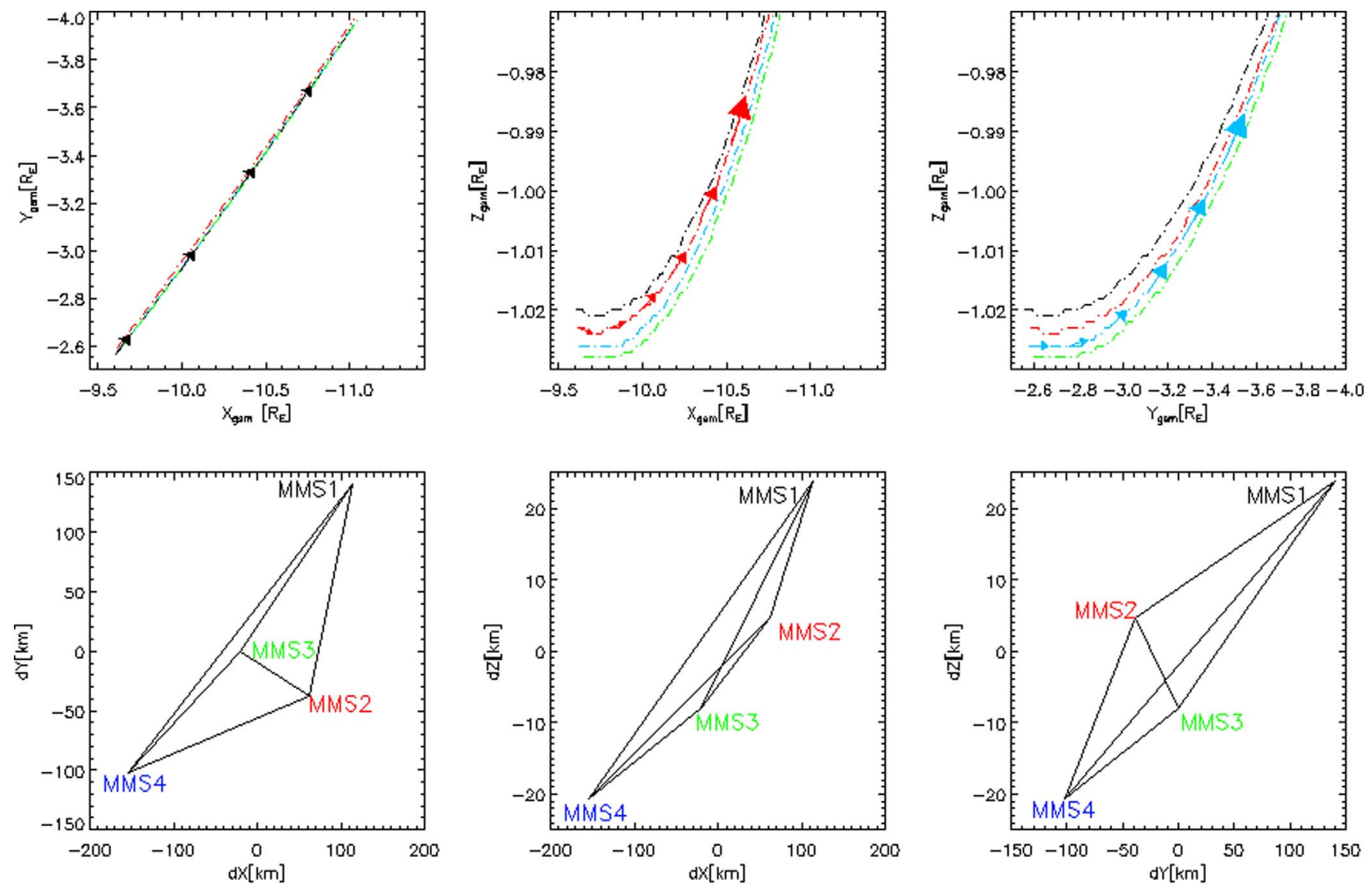

Figure 4. Orbits of the four MMS satellites (MMS1: black; MMS2: red; MMS3: green; MMS4: blue) between 02:16 and 03:36 UT on 2017 April 19 in GSE coordinates, with the arrow indicating their moving directions in the $X Y, X Z$, and $Y Z$ plane.

the local plasma heating. The high-quality data from four MMS satellites are available during these periods; thus, we investigate the potential heating mechanism such as plasma vortices and current enhancement in the following.

Before analyzing the plasma vortices, we plot the configuration of the four satellites during these periods in Figure 4. The top panels give moving orbits of the four satellites (MMS1: black; MMS2: red; MMS3: green; MMS4: blue) between 02:16 and 03:36 UT on 2017 April 19 in GSM coordinates, with the arrows indicating their moving direction in the $X Y, X Z$, and $Y Z$ plane. The bottom panels show their corresponding separations. The satellites were moving tailward in the magnetotail from distances larger than $9 R_{\mathrm{E}}$, away from Earth in the $X$-direction with a maximum separation of $268 \mathrm{~km}$ and a minimum separation of $51 \mathrm{~km}$ between them. They were located dawnward in the $Y$-direction with a maximum separation of $242 \mathrm{~km}$ and a minimum of $38 \mathrm{~km}$. They were moving from southward in the $Z$-direction with a maximum separation of $45 \mathrm{~km}$ and a minimum of $13 \mathrm{~km}$. In general, the separations between the four satellites were smaller than the distances among previous multisatellites in the terrestrial magnetotail. However, the three components of the plasma flows observed by FPI instruments on each of the four MMS satellites were different. We show the three components of the plasma flows measured by the four satellites in Figure 5.

\section{Characteristics of Earthward High-speed Flow}

Due to the small separations of the four satellites, the three components of the plasma flows measured by MMS were very similar to those shown in panels (a)-(d) of Figure 5. Therefore, we assume that the plasma flow or magnetic field data are linearly varying across the volume defined by the four MMS spacecraft. Different from the previous vortex-like flow using observations of a single satellite or less than four satellites, we combine the orbit data of the four satellites with their measured flow field to directly calculate the vorticity using the curlometer method as shown in Figure 5(e). From the separation of the four satellites given in Figure 4, the largest separation was in the $X$-direction, the middle was in the $Y$-direction, and the smallest was in the $Z$-direction. Three components of the plasma flow observed by the four satellites exhibited roughly a similar pattern. However, they were actually different in amplitude and even in direction. During the first event, directions of the three components of the plasma flow changed frequently; vorticity was obviously larger than that before $t 1$, but there was no significantly dominant component of vorticity. During the second event, directions of the three components of the high-speed flow changed relatively infrequently, and the obvious dominant component was in the $X$-direction, accompanied by comparatively large $Y$ and $Z$ components. Combining the flow field with the spatial separation, two terms of the vorticity in the $X$-direction $\frac{\partial V_{y}}{\partial z}-\frac{\partial V_{z}}{\partial y}$ were roughly equivalent, two terms of the vorticity in the $Y$-direction $\frac{\partial V_{z}}{\partial x}-\frac{\partial V_{x}}{\partial z}$ were mainly controlled by $\frac{\partial V_{x}}{\partial z}$, and two terms of the vorticity in the $Z$-direction $\frac{\partial V_{x}}{\partial y}-\frac{\partial V_{y}}{\partial x}$ were mainly controlled by $\frac{\partial V_{x}}{\partial y}$. The detailed results of three components and total vorticity $\left(W_{x}\right.$ : 


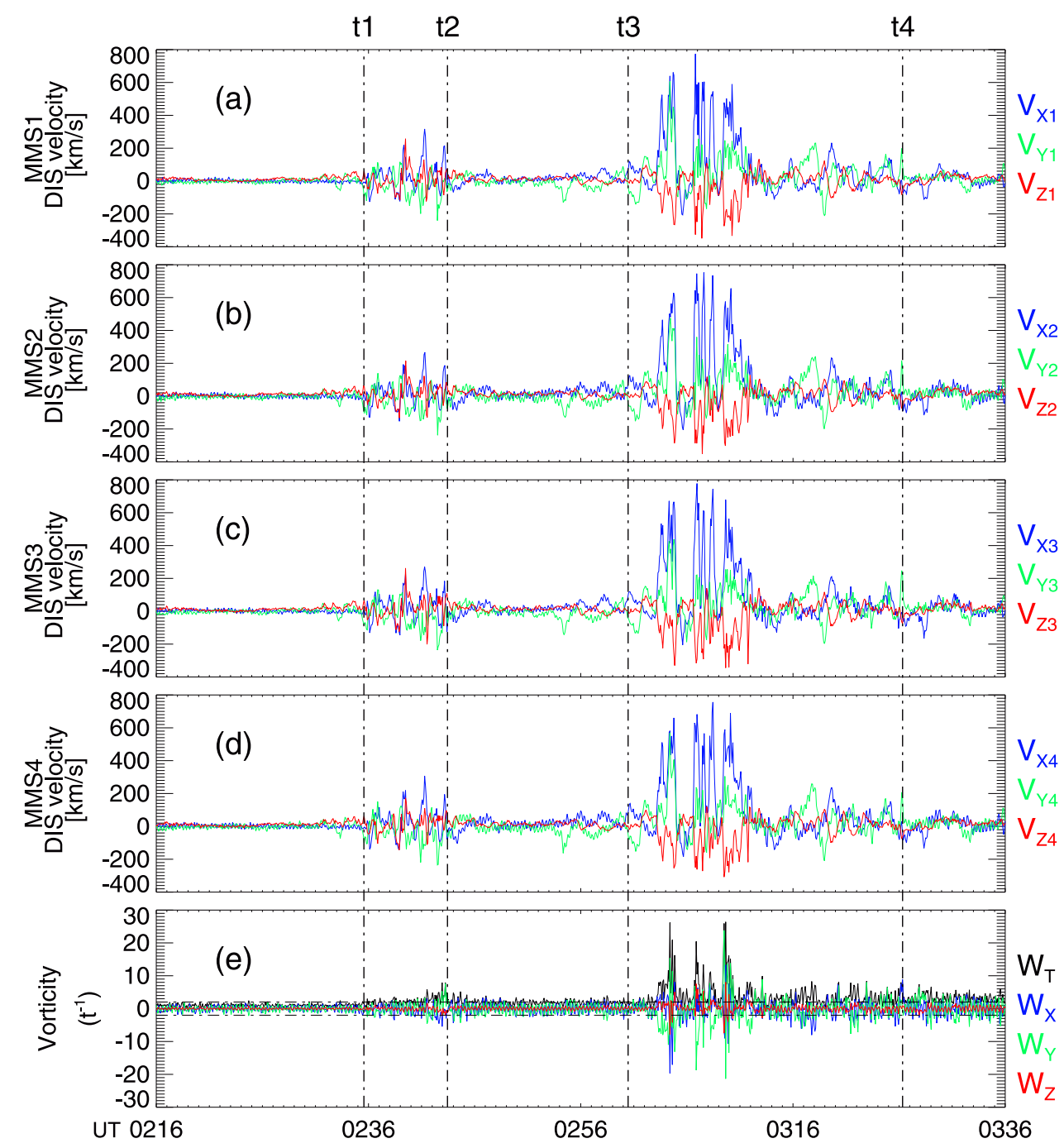

Figure 5. During the period between 02:16 and 03:36 on 2017 April 19, three components ( $V_{x}$ : blue; $V_{y}:$ green; $V_{z}:$ red) in GSE coordinates of the plasma flow from the four MMS satellites, (a) MMS1, (b) MMS2, (c) MMS3, and (d) MMS4. (e) The three components and their amplitude of vorticity ( $W_{x}$ : blue; $W_{y}:$ green; $W_{z}$ : red; $W_{T}$ : black), with the two horizontal black dotted lines representing $W= \pm 2 \mathrm{~s}^{-1}$. The vertical dashed lines (black) show the same two time periods of the flow enhancements as in Figure 1.

blue; $W_{y}$ : green, $W_{z}$ : red; $W_{T}$ : black) are shown in Figure $5(\mathrm{e})$. In general, the total vorticity during the first event was small and dominated by the $Y$ component. The total vorticity during the second event was very large and also dominated by the $Y$ component. This is mainly caused by the dominant $V_{x}$ component and smallest spatial separation $\frac{\partial V_{x}}{\partial z}$. It is worth mentioning that a standard propagation of error analysis of just the statistical errors associated with the plasma flow that might enter into the computations suggests that a base $12 \%$ error in the cross product would not be out of line (Gurgiolo et al. 2010).

From previous results of simulation (Parashar \& Matthaeus 2016), plasma heating is correlated with vorticity and current density, but more strongly with vorticity. In order to investigate the possible roles vorticity and currents play in the plasma heating from observations, we also calculate the current during this period as shown in Figure 6 using the curlometer method with the magnetic fields from the four MMS spacecraft (Dunlop et al. 2018).

The total current $j_{t}$ was below $50 \mathrm{nA} \mathrm{m}^{-1} \mathrm{~m}^{-1}$, with a mean value of less than $15 \mathrm{nA} \mathrm{m}^{-1} \mathrm{~m}^{-1}$ before $\mathrm{t} 1$ as shown by the black line in Figure 6 (e). During the period from t1 to t2, the current obviously increased to a mean value above $50 \mathrm{nA} \mathrm{m}^{-1} \mathrm{~m}^{-1}$. The dominant component was initially $j_{X}$ and then $j_{y}$ as shown in Figure 6(e). The field-aligned current (FAC), that is, component $J_{\text {bpara_nA }}$ (black) as shown in Figure 6(f), was swaying back and forth between parallel and antiparallel to the magnetic field. The currents were flowing into (antiparallel) the ionosphere and out (parallel) from the ionosphere. During the second period from $\mathrm{t} 3$ to $\mathrm{t} 4$, the total current $j_{t}$ obviously increased to a mean value above 50 $n A m^{-1} \mathrm{~m}^{-1}$ with a peak stronger than that of the first period. The dominant component was mostly $j_{y}$ and occasionally $j_{X}$ as shown in Figure 6(e). The FAC, that is, component $J_{\text {bpara_nA }}($ black) as shown in Figure 6(f), was swaying back and forth between parallel and antiparallel to the magnetic field. The magnitude of the divergence of magnetic field div $B \mid$, shown in Figure 6(f), was always below $0.4 \mathrm{nT} \mathrm{km}^{-1}$, even close to the ideal value of zero sometimes. The magnitude of curl $B \mid$, shown in Figure $6(\mathrm{~g})$, was comparable to and even larger than that of $|\operatorname{div} B|$, resulting in $Q=|\operatorname{div} B| /|\operatorname{curl} B|$, shown in Figure 6(i), being occasionally large. For most of the time, the magnitude of current $j_{t}$, shown by the black line in 


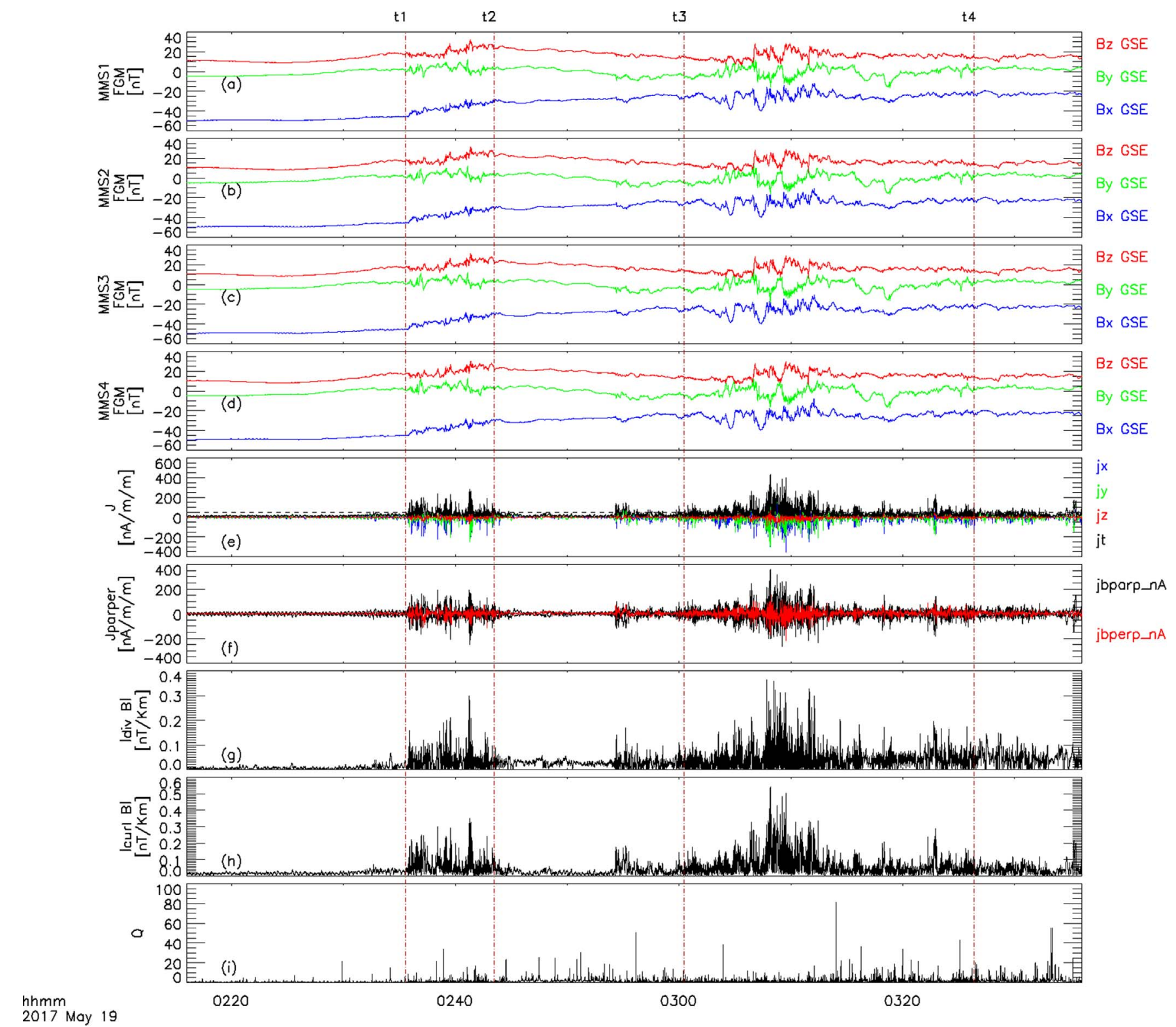

Figure 6. During the period between 02:16 and 03:36 on 2017 April 19, three components ( $B_{x}$ : blue; $B_{y}$ : green; $B_{z}:$ red) in GSE coordinates of the magnetic field from the four MMS satellites, (a) MMS1, (b) MMS2, (c) MMS3, and (d) MMS4. (e) The current determined using the curlometer method, $J_{x}$ : blue; $J_{y}$ : green; $J_{z}$ : red; $J_{t}$ : black (total current of $J_{x}, J_{y}$, and $J_{z}$ ). The horizontal dashed line represents $50 \mathrm{nA} \mathrm{m}^{-1} \mathrm{~m}^{-1}$. (f) Parallel (black) and perpendicular (red) components of the magnetic fields from the four MMS spacecraft. (g) $|\operatorname{div} B|$. (h) $|\operatorname{curl} B|$. (i) Ratio $Q=|\operatorname{div} B| / \mid$ curl $B \mid$, which gives an estimate of the quality of the curlometer current determination (Dunlop et al. 2018). The vertical dashed lines (red) show the same two time periods of the flow enhancements as in Figure 1.

Figure 6(f) during two periods, was above $50 \mathrm{nA} \mathrm{m}^{-1} \mathrm{~m}^{-1}$, indicating that the currents we estimate here are valid (Dunlop et al. 2018). The currents were flowing into (antiparallel) the ionosphere and out (parallel) from the ionosphere. In conjunction with Figure 5, we can find that plasma vortex structures were generally close to current structures.

In order to further check whether the plasma vortex structures and current density are intimately related to plasma heating and generation of anisotropies, we calculate correlations between multiple quantities of four MMS satellites for the two periods as shown in Table 1 . Here $\Delta T=T_{i}-\left\langle T_{i}\right\rangle$; the angle brackets $\langle\ldots\rangle$ denote a time average before the temperature increase before the two periods when the temperature was relatively stable at $t_{0}$ in the simulation (Parashar \& Matthaeus 2016). This expression $\triangle A_{p}=T_{i \perp} / T_{i \|}-1$ is for the proton anisotropy. It should be noted that our observations are $3 \mathrm{D}$, and the simulation was a purely $2 \mathrm{D}$, or nearly $2 \mathrm{D}$, geometry. Since the magnetic field in simulation is in the $X$ and $Y$-direction and does not include a guide field in the $z$ direction, the current and vorticity of $z$ components $\left(j_{z}, \omega_{z}\right)$ are mainly perpendicular to the field. The magnetic field in the observation is mainly in the $x$-direction (and $z$-direction), so we use $j_{\perp}, \omega_{\perp}$ to correspond approximately to $j_{z}, \omega_{z}$ in the simulation. We project the vorticity and current density into perpendicular to the magnetic field. $\omega_{\perp}$ is evaluated by $\omega$ cross unit vector of local magnetic field for each satellite separately. $j_{\perp}$ is evaluated by $\boldsymbol{j}$ cross unit vector of local magnetic field for each satellite separately.

As shown in Equation (7) of Yang et al. (2017b), energy exchange between fluid kinetic energy $E_{\alpha}^{f}$ of species $\alpha$ and the thermal (random) energy of the same species $\alpha E_{\alpha}^{\text {th }}$ took place only through the term $\left(\boldsymbol{P}_{\alpha} \cdot \nabla\right) \boldsymbol{u}_{\alpha}$. Here $\boldsymbol{P}_{\alpha}$ is the pressure 
Table 1

Correlations between Multiple Quantities of Four MMS Satellites for the Two Periods

\begin{tabular}{|c|c|c|c|c|c|c|c|c|}
\hline \multirow[b]{2}{*}{ Correlation } & \multicolumn{4}{|c|}{ Period from $\mathrm{t} 1$ to $\mathrm{t} 2$} & \multicolumn{4}{|c|}{ Period from $\mathrm{t} 3$ to $\mathrm{t} 4$} \\
\hline & mms1 & mms2 & mms3 & mms4 & $\mathrm{mms} 1$ & mms2 & mms3 & mms4 \\
\hline$\overline{\left(\omega_{\perp}, j_{\perp}\right)}$ & 0.0331 & 0.0288 & -0.0592 & 0.0389 & -0.0258 & -0.0258 & -0.0401 & 0.0414 \\
\hline$\left(\left|\omega_{\perp}\right|,\left|j_{\perp}\right|\right)$ & 0.0177 & 0.0255 & 0.0811 & -0.0114 & 0.1772 & 0.1801 & 0.2037 & 0.0754 \\
\hline$\left(\omega_{\perp}, \Delta T\right)$ & 0.5553 & 0.4499 & 0.4475 & 0.5045 & 0.2531 & 0.2529 & 0.1159 & 0.2847 \\
\hline$\left(\omega_{\perp}, \Delta A_{P}\right)$ & 0.1664 & 0.1576 & 0.2389 & 0.1127 & -0.1811 & -0.1755 & -0.2771 & -0.2063 \\
\hline$\left(\left|j_{\perp}\right|, \Delta T\right)$ & -0.2075 & -0.1686 & -0.0992 & 0.1253 & 0.0358 & 0.0445 & 0.0254 & -0.0112 \\
\hline$\left(\left|j_{\perp}\right|, \Delta A_{P}\right)$ & -0.1063 & -0.1051 & 0.0143 & 0.0353 & -0.0354 & -0.0391 & -0.0742 & 0.0177 \\
\hline
\end{tabular}

tensor, and $\boldsymbol{u}_{\alpha}$ is the fluid (flow) bulk velocity of species $\alpha$. The term of $-\left(\boldsymbol{P}_{\alpha} \cdot \nabla\right) \boldsymbol{u}_{\alpha}$ for protons/electrons in fully kinetic simulations (Yang et al. 2017a, 2017b) can be locally + or - . Calculation of the term $-\left(\boldsymbol{P}_{\alpha} \cdot \nabla\right) \boldsymbol{u}_{\alpha}$ for electrons could be negative or positive when parallel electron heating was observed by MMS (Chasapis et al. 2018); our calculation of the term $-\left(\boldsymbol{P}_{\alpha} \cdot \nabla\right) \boldsymbol{u}_{\alpha}$ for protons can be locally negative or positive when increases of perpendicular and parallel proton temperature were observed during the two periods. Considering that the negative of the term $-\left(\boldsymbol{P}_{\alpha} \cdot \nabla\right) \boldsymbol{u}_{\alpha}$ suggests a nearby source of energization, which resulted in the observed parallel heating, while at the point of observation, thermal energy is channeled into the flow as plasma expands and cools (Chasapis et al. 2018). We only calculate the correlations between multiple quantities of four MMS satellites for the two periods when the term $-\left(\boldsymbol{P}_{\alpha} \cdot \nabla\right) \boldsymbol{u}_{\alpha}$ is positive, which may suggest an increase of the thermal energy of the proton at the point where the MMS spacecraft is, pointing to a region of active electron heating.

The time correlation between signed $\left(\omega_{\perp}, j_{\perp}\right)$ for the four MMS satellites was close to positive/negative values of zero during the two periods, which is consistent with the result that the current sheets are typically accompanied by vorticity generated in a quadrupole configuration in the hybrid run (Parashar \& Matthaeus 2016). The time correlation between $\left(\left|\omega_{\perp}\right|,\left|j_{\perp}\right|\right)$ during the first period for the four MMS satellites was close to positive/negative values of zero. The time correlation between $\left(\left|\omega_{\perp}\right|,\left|j_{\perp}\right|\right)$ during the second period for MMS4 was close to positive values of zero, while the time correlation between $\left(\left|\omega_{\perp}\right|,\left|j_{\perp}\right|\right)$ during the second period for MMS1 and MMS2 had values close to 0.18 or even 0.20 for MMS3, which is slightly larger than the maximum of $Y$-axis of about 0.15 given in the top panel of Figure 6 for the hybrid run (Parashar \& Matthaeus 2016).

The time correlation between $\left(\omega_{\perp}, \Delta T\right)$ for MMS1 and MMS4 was slightly larger than 0.50 , with the other two smaller, about 0.45 during the first period, which is larger than the maximum of $Y$-axis of about 0.25 given in the bottom panel of Figure 6 for the hybrid run and is roughly consistent with the results shown by the blue line shown in the top panel of Figure 7 for PIC runs. The large correlation also illustrates that the four MMS satellites were located very close to the simulation center within $1 d_{i}$ during the first period. During the second period, the time correlation between $\left(\omega_{\perp}, \Delta T\right)$ for MMS4 was slightly larger than 0.28 , about 0.25 for MMS1 and MMS2, and slightly above 0.11 for MMS3, which is roughly consistent with the results shown by the blue line given in the bottom panel of Figure 6 for the hybrid run.

Considering that values appeared in more than one place in the top panel of Figure 7 for the PIC runs (Parashar \& Matthaeus 2016), we estimate that the relative locations of four MMS satellites were located within $2 d_{i}$ away from the simulation center during the first period from the time correlation between $\left(\omega_{\perp}, \Delta T\right)$. Compared with the results shown by the blue line in the bottom panel of Figure 6 for the hybrid run, time correlation between $\left(\omega_{\perp}, \Delta T\right)$ illustrates that the four MMS satellites were located within $10 d_{i}$ away from the simulation center during the second period.

We further analyzed the correlation between vorticity and proton temperature anisotropy $\triangle A_{p}=T_{i \perp} / T_{i \|}-1$. The time correlation between $\left(\omega_{\perp}, \triangle A_{p}\right)$ is positive, about 0.2 during the first period, again supporting that the four MMS satellites were located very close to the simulation center, within $1 d_{i}$ as shown by the blue line in the middle panel of Figure 7 for the PIC runs (Parashar \& Matthaeus 2016). The time correlation between $\left(\omega_{\perp}, \triangle A_{p}\right)$ is negative, about -0.2 during the second period, which is mainly due to the parallel anisotropy (negative $\triangle A_{p}$; Franci et al. 2016) that the increase of parallel temperature is larger than the perpendicular one shown by panel (c) of Figure 1.

In general, we can conclude that conditions of time correlation between $\left(\omega_{\perp}, \Delta T\right)$ and $\left(\omega_{\perp}, \Delta A_{p}\right)$ during the first period are generally similar to results for PIC runs, and those during the second period are generally similar to results for the hybrid simulation.

During the first period, amplitudes of the time correlation between $\left(\left|j_{\perp}\right|, \Delta T\right),\left(\left|j_{\perp}\right|, \triangle A_{p}\right)$ for MMS4 were much smaller than the time correlation between $\omega$ and corresponding quantities. The time correlation between $\left(\left|j_{\perp}\right|, \Delta T\right)$ is above 0.1 , again supporting that the MMS4 satellite was located very close to the simulation center, within $1 d_{i}$ as shown by the blue line in the middle panel of Figure 7 for the PIC runs (Parashar $\&$ Matthaeus 2016), which is very similar to what the green line shows in the top panel of Figure 7 for PIC runs. However, it is worth noting that the time correlations between $\left(\left|j_{\perp}\right|, \Delta T\right)$, $\left(\left|j_{\perp}\right|, \triangle A_{p}\right)$ for MMS1, MMS2, and MMS3 were in large amplitudes and their signs were negative. Results of observation for the first period are generally inclined to support that proton heating is more closely correlated with vorticity than current density close to the simulation center.

During the second period, amplitudes of the time correlation between $\left(\left|j_{\perp}\right|, \Delta T\right)$ for four MMS satellites were also much smaller than the time correlation between $\left(\omega_{\perp}, \Delta T\right)$. The time correlation between $\left(\left|j_{\perp}\right|, \Delta T\right)$ for MMS2 is above 0.04 and below 0.05 , which may indicate that MMS2 is located between 10 and $20 d_{i}$ away from the simulation center as shown by the green line in the bottom panel of Figure 6 for hybrid runs. The time correlation between $\left(\left|j_{\perp}\right|, \triangle A_{p}\right)$ for MMS4 is similar to what is shown by the green line in the middle panel of Figure 6 for hybrid runs, while the time correlations between $\left(\left|j_{\perp}\right|, \triangle A_{p}\right)$ for MMS1, MMS2, and MMS3 were in negative values. 
Results of observation for the second period are also generally inclined to support that proton heating is more closely correlated with vorticity than current density.

We further noticed that results of the time correlation $\left(\omega_{\perp}\right.$, $\Delta T$ ) during the first period were very similar to those given by the PIC simulation. Results of the time correlation $\left(\omega_{\perp}, \Delta T\right)$ during the second period were very similar to those for the hybrid run. The correlation between $\left(-\left(\boldsymbol{P}_{\alpha} \cdot \nabla\right) \boldsymbol{u}_{\alpha}, \Delta T\right)$ is slightly less than that between $\left(\omega_{\perp}, \Delta T\right)$ during the first period, while the correlation between $\left(-\left(\boldsymbol{P}_{\alpha} \cdot \nabla\right) \boldsymbol{u}_{\alpha}, \Delta T\right)$ is slightly larger than that between $\left(\omega_{\perp}, \Delta T\right)$ during the second period. A kinetic heating process that related to vorticity during the period may be just an accidental event; a direct energy transfer between flow and pressure can occur as a result not only of vorticity but also of the components of the shear tensor as has been measured in turbulence simulations (Del Sarto \& Pergaro 2018).

During the second period, the increase of temperature was accompanied by dominant earthward flow and positive $B_{z}$. This may suggest that the strong duskward electric field in earthward flow bursts with positive $B_{z}$ can be a very efficient accelerator in addition to vorticity during the second period.

\section{Discussion and Conclusions}

We have investigated the energy budget of earthward plasma flows and ion heating associated with flow vortices by direct calculation from measurements of four MMS satellites earthward of $-11 R_{\mathrm{E}}$ in the $X$-direction. The main results of our comprehensive examination of the energy budget, flow vortices, and ion heating are the following:

1. Between the starts and ends of both BBFs, the amplitude of energy flux densities could be ranked as $\varepsilon, U_{\mathrm{F}}, U_{\mathrm{R}}, U_{\mathrm{J}}$, and $U_{\mathrm{A}}$ in the periods from $\mathrm{t} 1$ to $\mathrm{t} 2$ and from $\mathrm{t} 3$ to $\mathrm{t} 4$. The largest is $U_{\mathrm{F}}$ in our two periods, which is a little different from the statistical average results (e.g., Østgaard et al. 2002a, 2002b). The obvious enhancement of ion temperature leads us to the conclusion that the energy density transported by high-speed earthward flows might be associated with ion heating other than the energy increase of the ring current, the Joule heating, and particle precipitation energy flux.

2. By means of the plasma flows simultaneously measured by four satellites of the MMS mission (Pollock et al. 2016), vorticity was directly calculated and is clearly larger than those times when the flow is nearly stagnant before $\mathrm{t} 1$. Large vorticities for the second period were mainly caused by the dominant $V_{x}$ component and smallest spatial separation $\frac{\partial V_{x}}{\partial z}$, which might be earlier than the simulation conditions that the earthward flow channel splits into two directions, and each of them deflects toward dawnside and duskside favoring the generation of the Region 1-sense FAC (Birn et al. 2011).

3. Our comparison of observation obtained by multisatellite measurements with simulations reveals some detailed and new information, in addition to some results consistent with previous simulations of the PIC run (Parashar \& Matthaeus 2016).

Plasma vortex structures are generated very close to current structure, which is consistent with previous results of simulations for the two periods. Their time correlation is positive for the first period from $\mathrm{t} 1$ to $\mathrm{t} 2$, which is different from previous simulations. Their time correlation, however, is weak and very close to zero for the second period, which is very similar to the previous simulation. Further investigation of time correlation between temperature and vorticity and current demonstrates that proton heating is more closely correlated with vorticity than current density for the first period, while they are found to be relatively correlated for the second period. Comparing the correlation obtained by observation and simulation, we suggest that the different correlations for the two periods might be caused by their different locations. We speculate that the four MMS satellites were located very close to the simulation center for the first period, since the results are roughly consistent with the position of the blue line shown in the top panel of Figure 7 (Parashar \& Matthaeus 2016). However, the spacecraft might be at a distance farther than $1 d_{i}$ and closer than $2 d_{i}$ away from the simulation center for the second period. The strong duskward electric field in earthward flow bursts with positive $B_{z}$ (Schödel et al. 2001; Takada et al. 2006) can be a very efficient accelerator for more significant temperature increases in addition to vorticity.

Time correlations of vorticity and proton anisotropy suggest that our speculation on the locations of four MMS satellites is relatively accurate. The magnitudes of time correlations of current and proton anisotropy are also consistent with our speculation of the locations (Parashar \& Matthaeus 2016). Although time correlation between $\left(\left|j_{\perp}\right|, \Delta T\right)$ for MMS4 is positive and consistent with the result of hybrid simulations during the first period, similar amplitudes but in negative sign of time correlation between $\left(\left|j_{\perp}\right|, \Delta T\right)$ for MMS1, MMS2, and MMS3 have not appeared for the hybrid run. This inconsistency indicated that comparison of satellite observations with the numerical results and theoretical models for 2D incompressible MHD was not easy.

It should be noted that the evaluated energy increase of the ring current (such as the increase of $\mathrm{O}^{+}$), Joule heating, and particle precipitation energy flux (Østgaard et al. 2002a) all might be caused by sources other than the energy transported by the plasma flow in the magnetotail. We have reasons to expect there should be more energy than the difference between the energy transported by the plasma flow and the energy increases in the ring current; the Joule heating and the particle precipitation energy flux results in either plasma heating or enhancements of plasma temperature. Indeed, our time correlation analyses of vorticity proton anisotropy, based on simultaneous measurements of four satellites, well support the concept that the conversion of larger fluid scale energy into thermal degrees of freedom (e.g., Klimontovich 1997; Howes 2015) is achieved by plasma flow vorticity (e.g., Huba 1996; Markovskii et al. 2006) for the first period. Plasma flow vorticity contributed partially to the temperature increase for the second period; other components of the flow shear tensor may play very important roles (Markovskii et al. 2006; Yang et al. 2017b; Del Sarto \& Pergaro 2018) on the enhancements of the temperature.

Research is supported by NSFC grants 41821003, 41174141, and 41874193. I.J.R. is supported in part by STFC grant ST/ N0007722/1 and Natural Environment Research Council Highlight Topic Grant SWIGS grant NE/P017150/1. M.W.D. is also partly supported by NERC SWIGS grant NE/P016863/1 and by STFC in-house research grant ST/M001083/1. MMS data are available from https://lasp.colorado.edu/mms/sdc/. The geomagnetic data are from http://wdc.kugi.kyoto-u.ac.jp/dstae/ index.html, and the high-resolution solar wind data are 
from https://omniweb.gsfc.nasa.gov/html/ow_data.html. The Space Physics Environment Data Analysis Software (SPEDAS, spedas.org) was used for the data processing.

\section{ORCID iDs}

\section{J. Rae (iD https://orcid.org/0000-0002-2637-4786}

\section{References}

Akasofu, S.-I. 1981, SSRv, 28, 121

Angelopoulos, V., Baumjohann, W., \& Kennel, C. F. 1992, JGR, 97, 4027 Angelopoulos, V., Kennel, C., \& Coroniti, F. 1994, JGR, 99, 21257 Artemyev, A. V., Zhang, X.-J., Angelopoulos, V., et al. 2018, JGR, 123, 5625 Baker, D. N., Pulkkinen, T. I., \& Hesse, M. 1997, JGR, 102, 7159 Baumjohann, W., Paschmann, W. G. G., \& Cattell, C. A. 1989, JGR, 94, 6597 Beaujardière, O., Lyons, L. R., Ruohoniemi, J. M., et al. 1994, JGR, 99, 287 Birn, J., Nakamura, R., Panov, E. V., et al. 2011, JGRA, 116, A01210 Cao, J. B., Duan, J. T., Du, A. M., et al. 2008, JGRA, 113, A07S15 Cao, J. B., Ma, Y. D., Parks, G., et al. 2006, JGR, 111, A04206 Cao, J. B., Ma, Y. D., Parks, G., et al. 2013, JGRA, 118, A018351 Cao, J. B., Yan, C. X., Dunlop, M. W., et al. 2010, JGRA, 115, A08205 Chasapis, A., Yang, Y., Matthaeus, W. H., et al. 2018, ApJ, 862, 32 De Spiegeleer, A., Hamrin, M., \& Pitkänen, T. 2017, JGRA, 122, 7042 Del Sarto, D., \& Pegoraro, F. 2018, MNRAS, 475, 181

Del Sarto, D., Pegoraro, F., \& Califano, F. 2016, PhRvE, 93, 053203

Du, A. M., Tsurutani, B. T., \& Sun, W. 2011, JGRA, 116, A12215

Dungey, J. W. 1961, PhRvL, 6, 47

Dunlop, M. W., Haaland, S., Dong, X., et al. 2018, in Electric Currents in Geospace and Beyond, ed. A. Keiling, O. Marghitu, \& M. Wheatland (Hoboken, NJ: John Wiley \& Sons), 67

Franci, L., Hellinger, P., Matteini, L., Verdini, A., \& Landi, S. 2016, in SOLAR WIND 14: Proc. Fourteenth Int. Solar Wind Conf. 1720, ed. L. Wang et al. (New York: AIP), 040003

Fu, H. S., Cao, J. B., Cully, C. M., et al. 2014, JGRA, 119, 9089

Fu, H. S., Cao, J. B., Vaivads, A., et al. 2016, JGRA, 121, 1263

Fu, H. S., Khotyaintsev, Y. V., Vaivads, A., et al. 2013, NatPh, 9, 426

Fu, H. S., Xu, Y., Vaivads, V., \& Khotyaintsev, Y. V. 2019, ApJL, 870, L22
Gurgiolo, C., Goldstein, M. L., Viñas, A. F., et al. 2010, AnGeo, 28, 2187

Hamrin, M., Pitkänen, T., Norqvist, P., et al. 2014, JGRA, 119, 9004

Hau, L. N., Phan, T. D., Sonnerup, B. U. Ö., \& Paschmann, G. 1993, GeoRL, 20, 2255

Hau, L. N., \& Sonnerup, B. U. Ö. 1993, GeoRL, 20, 1763

Hones, E. W., Paschmann, G., Jr., Bame, S. J., et al. 1978, GeoRL, 5, 1059

Howes, G. 2015, RSPTA, 373, 20140145

Huba, J. D. 1996, GeoRL, 23, 2907

Keiling, A., Angelopoulos, V., Runov, A., et al. 2009, JGRA, 114, A00C22

Kiehas, S. A., Runov, A., Angelopolos, V., et al. 2018, JGRA, 123, 1767

Klimontovich, Y. L. 1997, PhyU, 40, 21

Liu, C. M., Fu, H. S., Vaivads, A., et al. 2018, GeoRL, 45, 556

Liu, C. M., Fu, H. S., Xu, Y., et al. 2017, JGRA, 122, 594

Lu, S., Artemyev, A. V., Angelopoulos, V., et al. 2017, JGRA, 122, 8295

Ma, Y. D., Cao, J. B., Nakamura, R., et al. 2009, JGRA, 114, A07215

Markovskii, S. A., Vasquez, B. J., Smith, C. W., et al. 2006, ApJ, 639, 1177

Østgaard, N., Germany, G., \& Stadsnes, J. 2002a, JGRA, 107, 1233

Østgaard, N., Vondrak, R. R., \& Gjerloev, J. W. 2002b, JGRA, 107, 1246

Pang, X. X., Cao, J. B., Liu, W. L., et al. 2015, JGRA, 120, 2014JA020855

Parashar, T. N., \& Matthaeus, W. H. 2016, ApJ, 832, 57

Parks, G. K. 2004, Physics of Space Plasma, An introduction (Boulder, CO: Westview Press)

Pollock, C. J., Moore, T., Jacques, A., et al. 2016, SSRv, 199, 331

Pytte, T., McPherron, R. L., Hones, E. W., et al. 1978, JGR, 83, 663

Retinò, A., Sundkvist, D., Vaivads, A., et al. 2007, NatPh, 3, 236

Russell, C. T., Anderson, B. J., Baumjohann, W., et al. 2016, SSRv, 199, 189

Saur, J., Politano, H., Pouquet, A., \& Matthaeus, W. H. 2002, A\&A, 386, 699

Schödel, R., Nakamura, R., Baumjohann, W., \& Mukai, T. 2001, JGR, 106, 8381

Sergeev, V. A., Pellinen, R. J., \& Pulkkinen, T. I. 1996, SSRv, 75, 551

Sharma, P., McCourt, M., Quataert, E., \& Parrish, I. J. 2012, MNRAS, 420, 3174

Shiokawa, K., Baumjohann, W., \& Haerende, G. 1997, GeoRL, 24, 1179

Takada, T., Nakamura, R., Baumjohann, W., et al. 2006, GeoRL, 33, L21109

Tian, A. M., Zong, Q. G., Wang, Y. F., et al. 2010, JGRA, 115, A09204

Xu, Y., Fu, H. S., Liu, C. M., \& Wang, T. Y. 2018, ApJ, 853, 11

Yang, Y., Matthaeus, W. H., Parashar, T. N., et al. 2017a, PhPl, 24, 072306

Yang, Y., Matthaeus, W. H., Parashar, T. N., et al. 2017b, PhRvE, 95, 061201

Yu, Y., Cao, J. B., Fu, H. F., et al. 2017, JGRA, 122, 6139 